\title{
Apertura comercial y desindustrialización: un análisis de panel para América del Sur.
}

\section{Commercial opening and deindustrialization: A panel analysis for South America.}

Darío Hurtado

Universidad Internacional del Ecuador, Ecuador

Autor para correspondencia: cehurtadocu@uide.edu.ec

Fecha de recepción: 20 de junio de 2018 - Fecha de aceptación: 30 de agosto de 2018

Resumen: La desindustrialización es considerada como la reducción sostenida de la actividad industrial y como resultado puede conducir a una contracción de la demanda laboral en este sector. Sin embargo, es indispensable destacar que la desindustrialización, dependiendo del nivel de desarrollo económico al que haya llegado una economía, puede ser positiva o negativa. En los países desarrollados la desindustrialización no es un fenómeno negativo, sino que es una consecuencia natural de un mayor crecimiento y por ende un aumento en la productividad industrial, desplazando de tal modo, el trabajo liberado al sector de los servicios. En el caso de Latinoamérica, específicamente en el Sur, este fenómeno es prematuro y puede ocasionar graves repercusiones en los sistemas económicos, por tal motivo, está investigación centró su interés en analizar mediante un panel de datos la contribución de la apertura comercial - entre otros causantes - en la desindustrialización. Por otro lado, a las modelaciones obtenidas se les aplicaron los test del: multiplicador de LaGrange, Hausman, F, Wooldridge, Wald, Breusch - Pagan LM, con la finalidad de conseguir resultados fiables. Posteriormente, la evidencia empírica permitió aceptar la hipótesis planteada acerca de la influencia de la apertura comercial sobre la desindustrialización de los países de América del Sur, siendo esta variable estadísticamente significativa en las regresiones propuestas, concluyendo en consecuencia que, ante aumentos en la apertura comercial, el empleo en el sector industrial disminuye.

Palabras clave: desindustrialización; empleo industrial; apertura comercial; productividad industrial

Abstract: Deindustrialization is considered as the sustained reduction of industrial activity and as a result can lead to a contraction of labor demand in this sector. However, it is essential to emphasize that deindustrialization, depending on the level of economic development that an economy has reached, can be positive or negative. In developed countries deindustrialization is not a negative phenomenon, but is a natural consequence of higher growth and therefore an increase in industrial productivity, thereby displacing the work released to the service sector. In the case of Latin America, specifically in the South, this phenomenon is premature and can cause serious repercussions in the economic systems, for this reason, this research focused its interest in analyzing through a data panel the contribution of commercial openness - among others causes in deindustrialization. On the other hand, the obtained models were applied the multiplier tests of LaGrange, Hausman, F, Wooldridge, Wald, Breusch - Pagan LM, in order to obtain reliable results. 
Subsequently, the empirical evidence allowed accepting the hypothesis raised about the influence of trade liberalization on the deindustrialization of the countries of South America, this variable being statistically significant in the proposed regressions, concluding that, in view of increases in trade liberalization, employment in the industrial sector decreases. Key Words: deindustrialization; industrial employment; commercial opening; industrial productivity

\section{Introducción}

La dinámica del crecimiento económico mundial ha provocado fluctuaciones y variaciones importantes en los modos de producción y estructuras productivas de las sociedades a través de la historia; bajo este contexto, las economías en el periodo preindustrial se basaban fundamentalmente en la agricultura ocupando de este modo una cantidad significativa de la población para esta actividad ocasionado por la baja productividad laboral; en un segundo plano se encontraba la industria, la cual durante este periodo era de carácter artesanal y utilizaba fuentes de energía humana, animal, hídrica o eólica para hacer funcionar las maquinas. Posteriormente en el siglo XIX, en Inglaterra se da un importante acontecimiento conocido como Revolución Industrial (invención de motores a vapor, etc.) lo cual supone el tránsito de una economía agraria y artesanal a una economía basada en la industria y producción mecanizada, de tal modo que los campesinos pasaron a formar parte de las nóminas de las fábricas.

En la segunda mitad del siglo XX el crecimiento industrial en las economías desarrolladas (occidentales) fue tornándose negativo, presentando así mismo reducciones sostenidas en la demanda de empleo en este sector y aumentándose simultáneamente en el sector servicios. Rowthorn \& Ramaswamy (1997) explican que este fenómeno se debe al hecho de que la productividad laboral ha crecido más lentamente en los servicios que en la industria, elevando de este modo el precio de los servicios y haciendo las manufacturas relativamente más baratas. Igualmente concluyen que, la desindustrialización no es un fenómeno negativo sino una consecuencia natural de un mayor crecimiento en las economías avanzadas.

Bleaney \& Castilleja (2007), Dinopoulos \& Segerstrom (2007), Frenkel \& Rapetti (2011), Bleaney \& Tian (2012), Baró (2013), entre otros, han abordado este fenómeno considerando la clasificación entre países del Norte (desarrollados) y del Sur (en desarrollo). Los resultados obtenidos por los autores enunciados son significativos y muestran que la integración de los países del Sur al mercado del Norte ha provocado pérdidas en la demanda de mano de obra en las industrias de los países desarrollados.

Por otro lado, es importante destacar que este suceso ocurrido en el Norte está afectando de igual forma a países Latinoamericanos; tal como lo matizan las investigaciones realizadas por Escaith (2006), Vera (2009), Frenkel \& Rapetti (2011) entre varios; por tanto, el objetivo general de la presente investigación es abordar el proceso de desindustrialización a partir de la influencia que tiene la apertura comercial en el empleo de las economías de Sur América.

Simultáneamente, se pretende determinar el rol de la productividad dentro del cambio en los patrones de empleo industrial; por tanto, como objetivo específico se tiene: evaluar la influencia de la productividad laboral industrial dentro de los procesos de desindustrialización de las economías mencionadas previamente. En este caso, para hacer frente a esta propuesta, 
partimos de la siguiente hipótesis: "La desindustrialización es causada por la apertura comercial", con ello se determinará si efectivamente existe y es estadísticamente significativa la relación entre el empleo y la apertura comercial.

\section{Desindustrialización: Una visión general.}

La desindustrialización es considerada en la literatura como una disminución sostenida y progresiva de la participación laboral en los procesos de producción industrial, estando presente por lo general en economías de mercado industrializadas que han adquirido un nivel de desarrollo elevado. Igualmente, es necesario recalcar que la desindustrialización también puede evidenciarse por la reducción de la participación del valor agregado industrial sobre el PIB.

Con respecto al tema, Rowthorn et. al., suponen que en el transcurso del desarrollo económico prácticamente todas las economías desarrolladas siguieron una trayectoria similar en términos generales. Es decir que, en el periodo preindustrial las economías se basaban fundamentalmente en el excesivo uso de mano de obra para la agricultura debido a su baja productividad; en lo posterior, con el desarrollo económico en marcha la participación de la agricultura en el empleo nacional cayó y dio paso a un rápido aumento en la participación de las manufacturas (industrialización). Actualmente la participación laboral manufacturera está en declive y a la par existe un aumento de la participación de los servicios en el empleo nacional; por tanto, por analogía con la fase anterior este proceso es a menudo descrito como desindustrialización.

En relación a la fase de industrialización, Rowthorn \& Ramaswamy $(1997,1998)$ consideran dos factores que pueden dar explicación al cambio desde el sector agrícola al industrial. El primero se refiere a la ley de Engels la cual se refiere que al incrementarse el ingreso per cápita la proporción de ingreso gastado en productos agrícolas disminuye y aumenta el consumo de productos manufacturados y servicios (lado de la demanda). El segundo sobre el lugar de la oferta, es el rápido crecimiento de la productividad laboral en la agricultura debido a las innovaciones. Al combinar estos efectos de oferta y demanda, dan como resultado un gran movimiento de empleo desde la agricultura a la industria aumentando de tal modo el trabajo en las manufacturas y un descenso significativo de trabajo en la agricultura.

En lo referente a la fase de desindustrialización, Rowthorn \& Wells (1987) la clasifican como positiva, negativa y de cambio estructural. La desindustrialización positiva es recurrente únicamente en los países que han alcanzado un elevado nivel de desarrollo, con un PIB per cápita superior al promedio mundial, pleno empleo, alta competitividad industrial, sector servicios en crecimiento entre otros; la desindustrialización negativa se da en cambio, en economías en donde el dinamismo industrial esta normalmente acompañado de altos porcentajes de empleados en manufactura y muestra un desarrollo económico pobre; en este sentido, cuando la industria inicia a reducir personal, ya sea por descenso de la producción o un incremento de la productividad, este excedente no es reabsorbido por el sector servicios; por tanto, el desempleo crece y el ingreso se mantiene bajo. Finalmente, la desindustrialización por cambio estructural es cuando el patrón de exportaciones netas cambia desde las manufacturas hacia otros bienes y servicios, transfiriéndose así trabajo y recursos desde las manufacturas hacia otros sectores declinándose de esta manera el porcentaje de trabajo en la industria. 
En un estudio posterior, Rowthorn \& Coutts (2004) señalan como causantes de desindustrialización a:

- La Especialización. Ciertas actividades como el diseño, la restauración y el transporte que se realizaron previamente en el local por las empresas manufactureras se realizan cada vez más por los proveedores de servicios especializados. Esto representa una reclasificación en lugar de una verdadera contracción en el sector manufacturero.

- El Consumo. Los patrones de consumo en los países desarrollados cambian debido a aumentos de su ingreso (leyes de Engels); es decir, ocurre algo similar a lo descrito anteriormente para el caso de la agricultura.

- La Productividad. Por definición, la tasa de crecimiento de la productividad del trabajo es igual al crecimiento tasa de producción menos la tasa de crecimiento del empleo. Por lo tanto, si la salida en dos sectores es aumentando al mismo ritmo, el sector con el crecimiento más rápido de la productividad tendrá el menor crecimiento del empleo y viceversa. El porcentaje de empleo en el sector más dinámico se reducirá.

- El Comercio internacional. La competencia de las importaciones también puede aumentar la productividad mediante la eliminación de las actividades de bajo valor agregado o las empresas ineficientes.

- La Inversión. El gasto de inversión está sesgado hacia los bienes manufacturados, como maquinaria y materiales de construcción, por lo que una mayor tasa de inversión aumentará la participación de las manufacturas en la demanda total, y de ese modo aumenta la participación del sector manufacturero en la producción real y el empleo.

Finalmente, este proceso de desindustrialización que acarrea reducciones laborales progresivas en el sector manufacturero es considerado por Singh (1997), Frenkel \& Rapetti (2011) y Baró (2013) entre otros, como un síntoma del fracaso económico, siendo de esta manera para ellos primordial una política que detenga o revierta ese proceso. Contrario a esta posición Rowthorn \& Wells (1987), Rowthorn \& Ramaswamy (1997, 1998), Rowthorn \& Coutts (2004) y Tregenna (2011) entre otros investigadores, consideran que la reducción de empleo industrial es una característica normal del crecimiento económico; por tanto, para ellos lo primordial es que existan políticas que faciliten esta transición hacia una nueva estructura económica.

\section{Desindustrialización Norte - Sur.}

El fenómeno de desindustrialización ha despertado gran interés en la comunidad científica debido al impacto que ha tenido sobre el empleo y el cambio de las estructuras productivas en las economías desarrolladas. Los estudios que han abordado este tema generalmente han sido mediante la clasificación entre países del Norte y países del Sur, considerando elementos tales como la productividad, ingreso y especialización como factores internos y al comercio e inversión como factores externos.

En relación a este tema, Krugman (1988) argumenta que "los costos fijos de entrada y salida de los mercados podría llevar a una apreciación en el tipo de cambio, lo cual induciría a las empresas nacionales a salir del mercado y a las empresas extranjeras a entrar debido a la reducción en competitividad en costos de los nacionales frente a los extranjeros". En un estudio posterior tomando como unidad de análisis a la economía estadounidense, Krugman (1996) encontró que las entradas masivas de capital a los EE.UU. en los años 80 tuvo como 
contrapartida una redistribución considerable de los recursos de los sectores transables, con esta nueva asignación afirma que se contrajo el empleo y que las exportaciones se vieron perjudicadas frente a las importaciones. Posteriormente encontró que los flujos de capital hacia los Estados Unidos cayeron drásticamente, lo que posiblemente produjo un fuerte descenso en la tasa de cambio real y un creciente déficit comercial.

Por su lado, Saeger (1997) con datos de la Organización para la Cooperación y Desarrollo Económico (OCDE), inicia su investigación planteándose lo siguiente: ¿El comercio con los países menos desarrollados tiene un impacto adverso sobre los trabajadores de las economías desarrolladas? En esa investigación el autor contrasta la reducción del empleo manufacturero de los países del Norte con el aumento del empleo en el mismo sector para los países del Sur, concluyendo a la par que existe relación entre la desindustrialización y la integración de los países del Sur a la economía global. Con respecto al mismo argumento; Saeger (1997), utilizando estimación en efectos fijos para un panel de datos de la OCDE encontró que las importaciones desde el Sur son estadísticamente significativas para predecir la participación del empleo en las manufacturas y en el valor agregado; igualmente, encontró que la relación entre el aumento de la integración Norte-Sur y la declinación en la participación del empleo en la OCDE son económica y estadísticamente significativos.

Continuando con el mismo tópico, Rowthorn \& Ramaswamy (1997), Rowthorn, KozulWright \& Akyüz (1997) consideran que el comercio Norte-Sur podría provocar una reducción laboral de la mano de obra no cualificada en las economías desarrolladas, aun siendo mínimas las importaciones provenientes desde el Sur; igualmente mencionan que, el aumento de la flexibilidad del mercado laboral no corregirá este problema y las medidas proteccionistas tales como la aplicación de las normas laborales en el Sur, reducirían la renta global, por tanto, las políticas del mercado de trabajo menos convencionales como los subsidios de empleo para los trabajadores no calificados ofrecen una respuesta más eficaz beneficiando a los trabajadores, tanto en el Norte y como en el Sur.

En un estudio posterior, de acuerdo a los resultados obtenidos Rowthorn \& Ramaswamy (1998) suponen que si las exportaciones hacia el Sur incrementan en un 1 por ciento del PIB esto induciría a un incremento del 1 por ciento en el empleo manufacturero; inversamente, si las importaciones desde el Sur aumentan a un 1 por ciento del PIB esto resultaría en una caída del 5,3 por ciento en el empleo manufacturero. A conclusiones similares llegaron Kucera \& Milberg (2003) al realizar un estudio para diez países pertenecientes a la OCDE. Estos investigadores encontraron que desde 1970 hasta 1990 el comercio mundial de manufacturas ha dado un balance negativo en el empleo manufacturero de los países desarrollados, atribuyendo principalmente estas pérdidas al comercio Norte-Sur.

Ouattara \& Strobl (2004), Bleaney \& Castilleja (2007), Frenkel \& Rapetti (2011), Bleaney \& Tian (2012), coinciden en el hecho de que la apreciación del tipo de cambio real podría perjudicar a la competitividad de las empresas y por tanto afectar su crecimiento potencial, tal como lo menciona Krugman. Igualmente se considera a los salarios como un posible causante de la desindustrialización de los países del Norte. En relación a este tema, Dinopoulos \& Segerstrom (2007) en su investigación hacen una distinción entre los niveles salariales de los países desarrollados (Norte) y los países en desarrollo (Sur), posteriormente 
sugieren que para mantener el pleno empleo de mano de obra en el Norte, los salarios relativos en las economías desarrolladas deben caer lo suficiente para suplir la pérdida de producción y compensar el empleo con un aumento de la ocupación en investigación y desarrollo (I+D).

Rowthorn \& Ramaswamy (1997) en un estudio realizado con datos de la Organización para la Cooperación y Desarrollo Económico (OCDE) encontraron que el empleo industrial como porcentaje del empleo total ha disminuido desde inicios de los 70 hasta mediados de los 90 en aproximadamente un 10\%. Rowthorn (2010, 2013) y Tregenna (2011) argumentan que esta reducción laboral está explicada principalmente por aumentos sistemáticos en la productividad manufacturera. Igualmente mencionan que el empleo no es lo mismo que la producción. En muchas economías avanzadas, la productividad de fabricación está aumentando rápidamente con el resultado de que este sector está produciendo una mayor producción con menos trabajadores, concluyendo que la desindustrialización en términos de empleo como regla no implica caída de la producción.

Similar a lo encontrado por Rowthorn et. al., Saeger (1997) indica que el crecimiento de la productividad manufacturera es una de las razones para la contracción del empleo en este sector, añadiendo a la par que el rápido crecimiento relativo de la productividad manufacturera implica que la participación de manufacturas en el valor agregado sea constante o incluso aumente mientras el empleo disminuye. Recientemente, Broadberry (2006) encontró que la productividad en el sector agrícola ha experimentado un rápido incremento en los países del norte desde 1950 hasta 1990; por lo cual, mediante este resultado explica que el cambio en la estructura económica de los países desarrollados se debe tanto a las variaciones en la productividad como en la elasticidad ingreso de la demanda de dichas economías.

Finalmente, es importante aclarar que el efecto del crecimiento de la productividad laboral sobre las manufacturas es ambiguo; dado que, el rápido crecimiento de la productividad en ese sector hace que las manufacturas sean relativamente más baratas y por tanto estimula la demanda de estos, sin embargo, existe menos trabajo requerido para este sector.

\section{Desindustrialización en América del Sur.}

Pieper (2000), Vera (2009, 2011), Cruz (2010), Frenkel \& Rapetti (2011) y Nassif et. al. (2013) entre otros, han realizado estudios en los cuales exponen que economías pertenecientes al Sur están atravesando por circunstancias similares a las de los países desarrollados, por tal motivo, el presente trabajo de investigación tiene el propósito el evaluar los efectos de la apertura comercial sobre la desindustrialización de los países de América del Sur. En este caso, es importante destacar que el empleo en la industria en esta región se ha visto disminuido, de 1991 al 2017, en alrededor de 2,32\% (porcentaje de acuerdo a la población económicamente activa); sin embargo, las economías de Bolivia, Brasil, Paraguay y Perú han experimentado incrementos sustanciales en el empleo del sector industrial (véase tabla 1).

\begin{tabular}{llll}
\multicolumn{4}{c}{ Tabla 1: Empleo Industrial (\% PEA) } \\
\hline & $\mathbf{1 9 9 1}$ & $\mathbf{2 0 1 7}$ & Variación \\
\hline Argentina & 32.9 & 23.3 & -9.6 \\
Bolivia & 20.5 & 22.4 & 1.9 \\
Brasil & 20.5 & 20.8 & 0.4 \\
Chile & 26.3 & 22.8 & -3.5 \\
\hline
\end{tabular}




\begin{tabular}{llll}
\hline Colombia & 20.9 & 19.4 & -1.5 \\
Ecuador & 19.4 & 18.5 & -0.8 \\
Paraguay & 18.5 & 19.5 & 1.0 \\
Perú & 13.9 & 15.9 & 2.0 \\
Uruguay & 30.2 & 19.9 & -10.2 \\
Venezuela & 26.1 & 23.3 & -2.8 \\
América del Sur & 22.9 & 20.6 & -2.3 \\
\hline
\end{tabular}

Fuente: Banco Mundial

El empleo industrial de Uruguay y Argentina son los mayormente afectados en la región con una reducción porcentual del 10.2 y 9.6 respectivamente. Al considerar a los países que tienen variaciones negativas en el empleo del sector secundario, se puede calcular un nivel promedio de reducción de alrededor de cinco puntos porcentuales. Este fenómeno lo considera Vera (2009, 2011), Frenkel \& Rappetti (2011) entre otros, como desindustrialización prematura, debido a que los países en estudio no han adquirido un nivel de madurez y desarrollo económico que sustente este cambio.

Este caso de desindustrialización presente en las economías del Sur se puede catalogar como un fenómeno "negativo" debido al hecho de que las economías latinoamericanas no han alcanzado un nivel de desarrollo económico relativamente aceptable (tal como lo menciona Rowthorn \& Wells - 1987); por tanto, esta desindustrialización prematura podría afectar gravemente a la economía en su conjunto tal como lo menciona Pieper (2000).

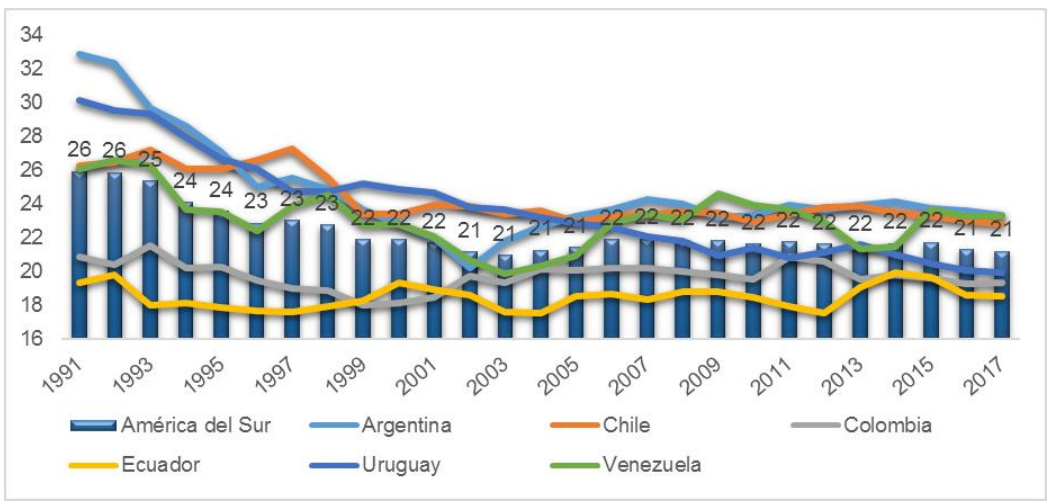

Figura 1. Variación del empleo industrial (\% PEA)

Fuente: Banco Mundial

Brady, Kaya \& Gereffi (2008) en su trabajo "Why is Latin America desindustrializing" consideran que la desindustrialización de América Latina es sorprendente dado que existen economías en similares circunstancias como las del Este de Asia que están industrializándose; en este sentido, consideran que varios factores jugaron en contra de los procesos industrializadores de la región tales como las dictaduras vividas durante ciertos periodos. Posteriormente, mencionan que la reducción laboral manufacturera en Latinoamérica podría estar relacionada con la rápida industrialización de China, sin embargo aclaran que ese proceso se venía dando desde mucho antes, pero que con la entrada de China a la Organización Mundial del Comercio este proceso se aceleró. 


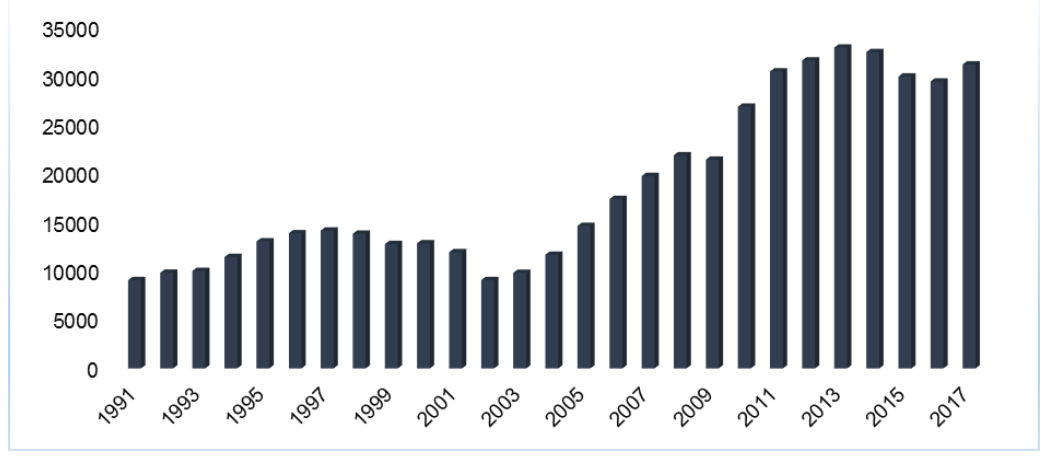

Figura 2: Productividad Industrial

Fuente: cálculos propios con datos de ILO, UNSTAT, OECD.

Ante estos sucesos Rowthorn y Ramaswamy (1997), Spilimbergo (1997), Elwell (2004), Clingingsmith \& Williamson (2004, 2005.a, 2005.b) y Dávalos (2007), explican que la desindustrialización puede ser causada, entre otros, por variaciones en la productividad de los sectores económicos. En el gráfico 2 se observa que la productividad industrial de un trabajador latinoamericano era aproximadamente de 25 mil dólares en 1980, sin embargo, para el 2010 esta se incrementó en un 250\% aproximadamente.

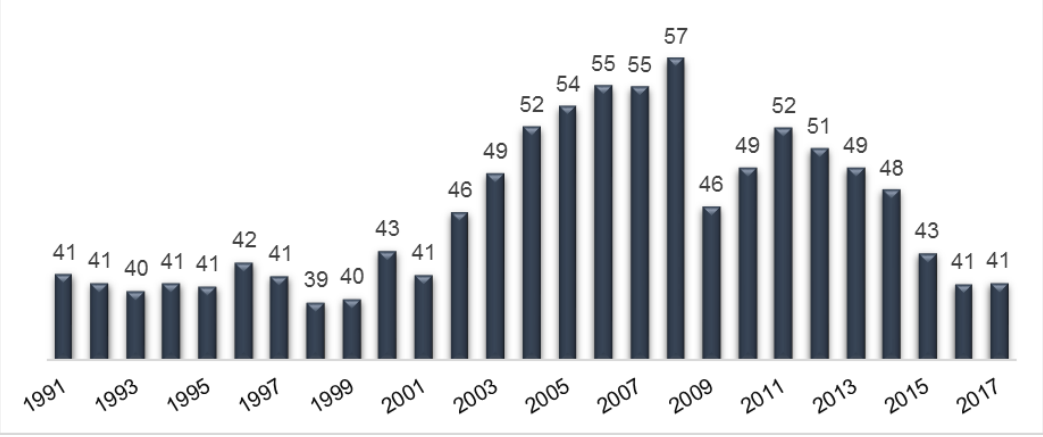

Figura 3: Apertura comercial

Fuente: cálculos propios con datos de ILO, UNSTAT, OECD.

La apertura comercial es un índice que indica cuán abierta esta una economía considerando para su cálculo las exportaciones e importaciones totales dividas para el PIB. Este indicador muestra que mientras más cercano este el índice a 1, más abierta esta la economía al mundo y mientras más cercano a 0 , indica que es una economía cerrada.

\section{Datos y métodos.}

La información que se obtuvo del Banco Mundial (WB), Organización Internacional de Trabajo (ILO) y Conferencia de las Naciones Unidas sobre Comercio y Desarrollo (UNCTAD; siglas en inglés); permitieron construir un conjunto de datos para analizar la influencia de la apertura comercial sobre el empleo industrial desde los años 1991 al 2017 en seis economías latinoamericanas.

Variable Dependiente.

El Empleo en la Industria de acuerdo al Banco Mundial corresponde a las divisiones 2-5 (CIIU, Revisión 2) o a las categorías de tabulación C-F (CIIU, Revisión 3), e incluye la 
explotación de minas y canteras (incluida la producción de petróleo), las industrias manufactureras, la construcción, y el suministro de servicios públicos (electricidad, gas y agua). Bajo este concepto, la variable dependiente utilizada para medir la desindustrialización es el empleo en la industria, la misma que es medida como porcentaje de la población económicamente activa (PEA). Esta variable ha sido construida con información proporcionada por la Organización Internacional del Trabajo (ILO) y la División de Estadísticas de las Naciones Unidas (UNSTAT).

\section{Variables Independientes.}

La productividad laboral es definida normalmente como la relación entre la producción obtenida por un sistema de producción o servicios y la cantidad de trabajo utilizado en el proceso productivo. La base de datos se construyó con información proporcionada por la UNSTAT, ILO y Banco Mundial. En este caso la Productividad Laboral Manufacturera fue obtenida utilizando la siguiente función:

$$
P T V I=\frac{V A I}{E M I}
$$

Dónde: PTVI es la Productividad Laboral Industrial (US\$ 1991), VAI corresponde al Valor Agregado Industrial (US\$ 1991) y EMI es el empleo en la Industria.

La apertura comercial es un indicador que se define como el porcentaje que suponen los flujos del comercio exterior sobre el total del PIB.

$$
A C=\frac{X+M}{P I B} * 100
$$

Dónde: AC es la apertura comercial, X son las exportaciones y M las importaciones. Este indicador muestra que una economía estará presente en los mercados internacionales cuanto mayor sea su grado de participación de la suma de las exportaciones más las importaciones en la producción nacional.

\section{Modelo.}

La metodología utilizada en la presente investigación se basa en un análisis econométrico desarrollado por Rowthorn \& Ramaswamy (1998). En este estudio los autores realizan un modelo simple de estimación usando datos de panel para 18 países industrializados sobre el periodo 1963-1994, para el cual obtuvo un total de 510 observaciones.

La ecuación a estimar es la siguiente:

$$
E M I=\gamma_{0}+\gamma_{1} A C+\gamma_{2}(A C)^{2}+\sum_{i>2} \propto_{i} Z_{i}+\text { error }
$$

La discusión anterior implica que los coeficientes deben tener los siguientes signos:

$$
\gamma_{1},>0 ; \gamma_{2}<0
$$


Dónde: EMI es la participación laboral en la industrial, AC es la apertura comercial y Z son las variables de control incluidas en el modelo. La ecuación 2 indica que el empleo industrial esta explicado por las variaciones en la apertura comercial en el corto y largo plazo.

\section{Estimación y discusión de resultados.}

De acuerdo a la información recolectada y al objetivo propuesto, en la presente investigación se hará uso de datos de Panel; por lo cual, es necesario determinar el método de Panel que se aplicara para la estimación de los resultados. El método más simple para analizar un panel de datos es omitiendo las dimensiones del espacio y el tiempo existentes en los datos agrupados y calcular de esta manera la regresión mediante $\mathrm{MCO}$; sin embargo, existen varios factores que no considera este procedimiento debido a que supone que el intercepto es el mismo para todas las unidades transversales.

Por otro lado, es probable que se requiera controlar el carácter individual de cada estado, suponiendo para ello que cada unidad transversal tiene un intercepto diferente. En este caso se requeriría utilizar el método de Efectos Aleatorios; por tanto, para corroborar el método a utilizar es preciso someter a prueba el método Pooled y el de Efectos Aleatorios.

\begin{tabular}{|c|c|c|c|c|}
\hline VARIABLES & POOLED & $\begin{array}{l}\text { FIXED } \\
\text { EFFECTS }\end{array}$ & $\begin{array}{l}\text { RANDOM } \\
\text { EFFECTS }\end{array}$ & $\begin{array}{l}\text { TWO WAY FIXED } \\
\text { EFFECTS }\end{array}$ \\
\hline Apertura comercial & $\begin{array}{l}-0.0452 * * \\
(0.0185)\end{array}$ & $\begin{array}{l}-0.107 * * * \\
(0.0188)\end{array}$ & $\begin{array}{l}-0.0867 * * * \\
(0.0189)\end{array}$ & $\begin{array}{l}-0.125 * * * \\
(0.0232)\end{array}$ \\
\hline $\begin{array}{l}\text { Productividad } \\
\text { industrial }\end{array}$ & $\begin{array}{l}4.41 \mathrm{e}-05^{*} \\
(2.39 \mathrm{e}- \\
05)\end{array}$ & $\begin{array}{l}-5.45 \mathrm{e}-05 * * * \\
(1.49 \mathrm{e}-05)\end{array}$ & $\begin{array}{l}-4.27 \mathrm{e}-05 * * * \\
(1.65 \mathrm{e}-05)\end{array}$ & $\begin{array}{l}-3.92 \mathrm{e}-05^{* * *} \\
(2.90 \mathrm{e}-05)\end{array}$ \\
\hline _Iyear_1992 & & & & $\begin{array}{l}-0.117 \\
(0.812)\end{array}$ \\
\hline _Iyear_1993 & & & & $\begin{array}{l}-0.715 \\
(0.813)\end{array}$ \\
\hline$:$ & & & & : \\
\hline $\begin{array}{l}: \\
\text { : }\end{array}$ & & & & : \\
\hline _Iyear_2016 & & & & $\begin{array}{l}-3.925 * * * \\
(1.004)\end{array}$ \\
\hline _Iyear_2017 & & & & $\begin{array}{l}-3.952 * * * \\
(1.034)\end{array}$ \\
\hline Constant & $\begin{array}{l}23.61 * * * \\
(0.883)\end{array}$ & $\begin{array}{l}28.27 * * * \\
(0.864)\end{array}$ & $\begin{array}{l}27.13 * * * \\
(0.979)\end{array}$ & $\begin{array}{l}31.46 * * * \\
(1.161)\end{array}$ \\
\hline Observations & 162 & 162 & 162 & 162 \\
\hline $\mathrm{R}$-squared & 0.045 & 0.265 & & 0.596 \\
\hline Rank & 3 & 3 & 3 & 29 \\
\hline r2_a & 0.0332 & 0.232 & & 0.492 \\
\hline $\mathrm{F}$ & 3.766 & 27.82 & & 6.740 \\
\hline Number of country & & 6 & 6 & 6 \\
\hline
\end{tabular}

En la tabla 2 se muestran los resultados de los diferentes modelos de panel utilizados en la investigación; sin embargo, es interesante aplicar el mejor modelo para determinar la influencia de la apertura comercial sobre la desindustrialización en los seis países en estudio. En 
ese contexto, se aplica el test del multiplicador de Lagrange de Breush y Pagan, siendo el resultado obtenido el siguiente:

Test: $\operatorname{Var}(\mathrm{u})=0$

chibar2 $(01)=604.41$

Prob $>$ chibar $2=0.0000$

El p-value de la prueba nos indica que es preferible utilizar la estimación de efectos aleatorios en lugar del método agrupado. Una vez conocido el resultado, se puede considerar otra manera de modelar el carácter individual de cada estado; es decir, suponiendo que las diferencias entre estados no son aleatorias sino constantes o fijas, para ello se utiliza el método de Efectos Fijos. En otras palabras, el modelo de Efectos Fijos considera que existe un término constante diferente para cada individuo y supone que los efectos individuales son diferentes entre sí; por otro lado, el modelo de Efectos Aleatorios considera que los efectos individuales no son independientes entre sí, sino que están distribuidos aleatoriamente alrededor de un valor dado. La prueba por excelencia para elegir el método es la denominada Prueba de Hausman; mediante este test, se plantea que los estimadores de efectos aleatorios y de efectos fijos no difieren sustancialmente. El resultado de la prueba es el siguiente:

Test: Ho: difference in coefficients not systematic

$$
\begin{aligned}
\operatorname{chi} 2(2) & (b-B)^{\prime}\left[\left(V_{-} b-V_{-} B\right)^{\wedge}(-1)\right](b-B) \\
& =151.40 \\
\text { Prob }>\text { chi } 2 & =0.0000
\end{aligned}
$$

La prueba de Hausman acusa un valor de p_chi $2<0.01$; por tanto, se rechaza la hipótesis nula de igualdad al 99\% de confianza y se asumiría las estimaciones mediante el método de efectos fijos. En este caso, la incorporación de variables dicotómicas estatales permite modelar las particularidades de las unidades transversales que no cambian en el tiempo pero que sí afectan el resultado de interés. Así mismo es posible agregar variables dicotómicas temporales que capturen eventos comunes durante un periodo u otro. Por tanto, para medir su relevancia se utiliza la prueba $\mathrm{F}$ para testear la significancia conjunta de las variables dicotómicas temporales presentes en el modelo. El resultado se presenta a continuación:

$$
\begin{aligned}
F(26,128) & =4.03 \\
\text { Prob }>F= & 0.0000
\end{aligned}
$$

El p-value para la prueba $\mathrm{F}$ es menor a 0.001 por tanto es posible afirmar que las variables dicotómicas temporales son conjuntamente significativas y pertenecen al modelo. Una vez obtenidos los resultados anteriores es necesario probar la existencia de correlación serial o autocorrelación en el modelo que se pretende analizar. Para testear este problema se hará uso de la prueba propuesta por Wooldridge y se plantea como hipótesis nula la no existencia de autocorrelación. El resultado se presenta a continuación:

H0: no first-order autocorrelation

$$
\begin{aligned}
\mathrm{F}(1, \quad 5) & =50.530 \\
\text { Prob }>\mathrm{F} & =0.0009
\end{aligned}
$$


El valor de F mostrado es estadísticamente significativo; por tanto, se rechaza la hipótesis nula de no autocorrelación de primer orden. En este caso existe un problema que podría ser solucionado mediante un modelo de efectos fijos con término autorregresivo de grado 1 (AR1) que controla por la dependencia de $t$ con respecto a $t-1$.

En tanto a la Heteroscedasticidad, la prueba por excelencia es el test Modificado de Wald y se testea la no existencia de heteroscedasticidad dentro del modelo. El resultado es el siguiente:

$$
\begin{aligned}
& \mathrm{H} 0 \text { : } \operatorname{sigma}(\mathrm{i})^{\wedge} 2=\operatorname{sigma}^{\wedge} 2 \text { for all } \mathrm{i} \\
& \operatorname{chi} 2(6)=102.27 \\
& \text { Prob }>\text { chi } 2=0.0000
\end{aligned}
$$

El p-value encontrado en el test es menor a 0.001; por tanto se rechaza la hipótesis nula de varianza constante. Finalmente, se debe testear la no existencia de correlación contemporánea y para ello se utilizara la prueba de Breusch y Pagan. Los resultados son los siguientes:

Breusch-Pagan LM test of independence: $\operatorname{chi} 2(15)=87.091, \operatorname{Pr}=0.0000$ Based on 27 complete observations over panel units

El p-valué del estadístico chi2 indica que se puede rechazar la hipótesis nula y se acepta la alternativa de que existe correlación contemporánea. Ahora bien, los problemas de correlación contemporánea, heteroscedasticidad y autocorrelación que se han estimado pueden solucionarse conjuntamente con estimadores de Errores Estándar Corregidos para Panel (Panel Corrected Standard Errors, PCSE); por tanto, el método de estimación será un modelo PCSE.

$$
\text { E. Industria }=23.98-0.026 \text { A. Comercial }-7.21 e^{-07} P . \text { Industrial }
$$

La ecuación cinco reporta los resultados encontrados mediante datos en panel y que dan sustento empírico a la implicación de la apertura comercial en la desindustrialización en las economías del sur de América. Los parámetros estimados se obtuvieron mediante el uso del método de PCSE, ya que ayuda a resolver problemas de correlación contemporánea, heterocedasticidad y autocorrelación, para un panel de datos creado para las 6 economías en estudio. Tal como se puede observar el resultado del efecto de la apertura comercial sobre el empleo manufacturero es estadísticamente significativa y los signos de sus parámetros están acordes a lo esperado (de acuerdo a lo propuesto por Rowthorn y Ramaswamy 1998).

\section{Conclusiones}

Con respecto al método de estimación, el modelo de Errores Estándar Corregidos para Panel (PCSE) seleccionado para las estimaciones del empleo manufacturero es el más adecuado para la presente investigación, debido a que ayuda a corregir los problemas de correlación contemporánea, heteroscedasticidad y autocorrelación presentes en el modelo de Efectos Fijos y de esta manera obtener valores más confiables.

La evidencia empírica de la presente investigación permite aceptar la hipótesis de que la apertura comercial y la productividad, juegan un rol relevante para explicar la desindustrialización en los países suramericanos. En este caso se pudo observar que estas 
variables son estadísticamente significativas y por tanto se puede afirmar que son determinantes de la desindustrialización prematura de la región en estudio.

\section{Bibliografía}

Baró, E. (2013). ¿Desindustrialización o Metamorfosis de la Industria? La nueva relación entre las actividades manufacturera y terciaria. Dialnet(387), 33-48.

Bleaney, M., \& Castilleja, L. (2007). Real Exchange Rate, Valuation Effects and Growth in Emerging Markets. Centre for Research in Economic Development and International Trade (CREDIT)(07/12), 1-23.

Bleaney, M., \& Tian, M. (2012). Exchange Rates and Trade Balance Adjustment a Multi-Country Empirical Analysis. Centre for Research in Economic Development and International Trade, 1-33.

Brady, D., Kaya, Y., \& Gereffi, G. (2008). Why is Latin America Deindustrializing? Deparment of Sociology, Duke University, 1-47.

Broadberry, S. (2006). Agriculture and Structural Change: Lessons from the UK experience in an international context. University of Warwick, 1-28.

Clingingsmith, D., \& Williamson, J. G. (2004). India's De-industrialization Under British Rule: New Ideas, New Evidence. National Bureau of Economic Research(10586), 1-40.

Clingingsmith, D., \& Willianson, J. G. (2005.a). India's Deindustrialization in the 18th and 19th Centuries. Harvard University, 1-46.

Clingingsmith, D., \& Williamson, J. G. (2005.b). Mughal Decline, Climate Change and Britain's Industrial Ascent: An Integrated Perspective on India's 18th and 19th Century Deindustrialization. National Bureau of Economic Reserch(11730), 1-56.

Coutts, K., Glyn, A., \& Rowthorn, R. (2007). Structural change under New Labour. Cambridge Journal Economics, 845-861.

Dávalos López, E. (2007). Deindustrialization in the United States. Center for Research on North America (CISAN), 29-32.

Dinopoulos, E., \& Segerstrom, P. (2007). North-South Trade and Economic Growth. University of Florida; Stockholm School of Economics, 1-37.

Elwell, C. K. (2004). Deindustrialization of the U.S. Economy: The Roles of Trade, Productivity and Recession. CRS Report for Congress, 1-27.

Escaith, H. (2006). Industrialización Truncada y Tercerización Sustitutiva en América Latina. Problemas del Desarrollo, 37(147).

Frenkel, R., \& Rapetti, M. (2011). Fragilidad Externa o Desindustrialización: ¿Cuál es la principal amenaza para América Latina? CEPAL(116), 1-37.

Kollmeyer, C. (2009). Explaining Deindustrialization: How Afluence, Productivity Growth and Globaliztion Diminish Manufacturing Employment. American Journals of Sociology, 114(6), 1644-1674.

Krugman, P. (1988). Deindustrialization, Reindustrialization and the Real Exchange Rate. National Bureau of Economic Research(2586), 1-32. 
Krugman, P. (1996). Domestic Distortions and the Deindustrialization Hypothesis. National Bureau of Economic Research(5473), 1-31.

Kucera, D., \& Milberg, W. (2003). Deindustrialization and Changes in Manufacturing Trade: Factor Content Calculations for 1978-1995. Review of World Economics / Weltwirtschaftliches Archiv, 139(4), 601-624.

Nassif, A., Feijó, C., \& Araujo, E. (2013). Structural Change and Economic Development: Is Brazil Catching up or Falling Behind? United Nations Conference on Trade and Development (UNCTAD), 1-23.

Ouattara, B., \& Strobl, E. (2004). Foreign Aid Inflows and the Real Exchange Rate in the CFA Franc Zone. Centre for Research in Economic Develpment and International Trade (CREDIT)(04/07), 1-23.

Piper, U. (2000). Deindustrialisation and the Social and Economic Sustainability Nexus in Developing Countries: Cross-Country Evidence on Productivity and Employment. Maastricht Economic Research Institute on Innovation and Technology, 66-99.

Rowthorn, R. (2010). Combined and Uneven Development: Reflections on the North-South Divide. Spatial Economic Analysis, 5(4).

Rowthorn, R., \& Coutts, K. (2004). De-industrialization and the Balance of Payments in Advanced Economies. United Nations Conference on Trade and Development(170).

Rowthorn, R., \& Coutts, K. (2013). De-Industrialisation and the balance of payments in advanced economies. Future of Manufacturing Project, 1-34.

Rowthorn, R., \& Ramaswamy, R. (1997). Deindustrialization: Causes And Implications. International Monetary Fund, 10(42), 1-38.

Rowthorn, R., \& Ramaswamy, R. (1998). Growth, Trade and Deindustrialization. International Monetary Fund(60), 1-28.

Rowthorn, R., \& Ramaswamy, R. (1999). Growth, Trade and Deindustrialization. IMF Staff Papers, 46(1), $18-41$.

Rowthorn, R., \& Wells, J. (1987). De industrialization and Foreign Trade. Cambridge University Press, 431.

Rowthorn, R., Kozul-Wright, R., \& Akyüz, Y. (1997). Adapting to North-South Trade a General Equilibrium Aproach to Policy Options. Oxford Economic Papers, 483-503.

Saeger, S. S. (1997). Globalization and Deindustrialization: Myth and Reality in the OECD. Review of Wolrd Economics / Weltwirtschaftliches Archives, 133, 579-608.

Spilimbergo, A. (1997). De-industrialization and Trade. International Monetary Fund, 1-37.

Tregenna, F. (2011). Manufacturing Productivity. Deindustrialization and Reindustrialization. World Institute for Development Economic Research(57).

Vera, L. (2009). Cambio Estructural, desindustrialización y pérdidas de productividad: evidencia para Venezuela. Cuadernos del CENDES, 89-115.

Vera, L. (2011). Paradojas de la desindustrialización: ¿hay evidencia de la tercera Ley de Kaldor para Venezuela? Cuadernos del CENDES. 